\title{
REFLEXIONES SOBRE LA FORMACIÓN DE DOCENTES DE LENGUA EXTRANJERA EN COLOMBIA
}

\section{REFLECTIONS ON THE FOREIGN LANGUAJE TEACHER FORMATION IN COLOMBIA}

\author{
Lizeth Ramos Acosta \\ Unidad Central del Valle del Cauca \\ Tuluá, Colombia
}

\section{RESUMEN}

Esta investigación histórica de carácter interpretativo basada en la comprensión de fenómenos sociales educativos según SáezRosenkranz (2016), se centró en el análisis del concepto de formación de los docentes de inglés en Colombia a la luz de la normatividad (leyes, decretos y planes) emanada por entes gubernamentales desde 1980 cuando se extiende la enseñanza del inglés en las instituciones educativas colombianas, hasta 2014 cuando se crea el Sistema Colombiano de Formación de Educadores y Lineamientos de Política. Los resultados ponen de manifiesto que, en el campo de la enseñanza de las lenguas extranjeras, la formación de los docentes ha estado marcada por otras nociones como el entrenamiento y la capacitación gracias a la influencia de organizaciones internacionales que imponen un concepto de formación que se aleja de los procesos de transformación de los maestros, la reflexión sobre su práctica y la comprensión de la disciplina que funda su labor docente.

Palabras clave: Formación, docentes, inglés, políticas públicas.

\section{ABSTRACT}

This historical interpretative investigation based on the understanding of social educational phenomena according to Saez-Rosenkranz (2016), focused on the analysis of the concept of formation of English teachers in Colombia in the light of the norms (laws, decrees and plans) aproved by government entities since 1980 
when English teaching in Colombian educational institutions is extended, until 2014 when the Colombian System for Educating Educators and Policy Guidelines was created. The results show that, in the field of foreign language teaching, teacher education has been marked by other notions such as teacher training, thanks to the influence of international organizations that impose a concept of teacher education that moves away from the processes of teacher transformation, reflection of their practicum and understanding of the discipline that constitues the base of teaching.

Key words: Formation, teachers, English, public policies.

\section{INTRODUCCIÓN}

La preocupación por la formación de docentes en Colombia surge a partir de la separación de la iglesia católica del contexto educativo. La escuela toma el lugar que durante siglos ocupó la iglesia y el Estado se convierte en el ente regulador y controlador de los procesos de enseñanza y aprendizaje de los maestros en el siglo XIX (Calvo, Rendón y Rojas, 2004).

Antes de la promulgación de la Ley 115 de 1994 , en la primera mitad del siglo $\mathrm{XX}$, la formación de los docentes se encontraba a cargo de dos grandes instituciones: las escuelas normales $y$, después de la década de los años cincuenta, las facultades de educación. Con la creación de las facultades de educación, se espera el mejoramiento de la calidad de la formación de los docentes que durante décadas estuvo a cargo de las escuelas normales. Posteriormente, en la década de los años sesenta, el gobierno de turno, favorece la proliferación de cursos de perfeccionamiento o especialización en las universidades. De esta manera, la formación de los docentes se concentró en el desarrollo de técnicas que le permitían al maestro aplicar el currículo desarrollado por agentes y agencias internacionales, desconociendo el contexto colombiano.

Recientemente, gracias a los procesos de globalización, internacionalización de la educación, movilidad de estudiantes y profesionales, se han generado transformaciones en la enseñanza de las lenguas extranjeras y los procesos de formación de los docentes de inglés en el país. Los discursos provenientes de agencias internacionales sobre certificación de suficiencia de la lengua y acreditación de programas académicos, han introducido cambios en los procesos de formación de los docentes de inglés, especialmente a partir de la década de los años ochenta, cuando no se requería ni suficiencia en la lengua ni poseer título de licenciado en lenguas para enseñar el idioma pues correspondía a maestros de otras áreas de conocimiento impartir dicha cátedra. Además, en las últimas décadas, la preocupación por la formación de los docentes se ha convertido en un eje fundamental en la aprobación de políticas públicas como estrategia para alcanzar las metas propuestas por el Ministerio de Educación Nacional (MinEducación) en materia de enseñanza y aprendizaje del inglés. Además, las recientes disposiciones gubernamentales sobre el bilingüismo en el país y su estrecha relación con los procesos de formación de los docentes de inglés, han sido el punto de partida para el planteamiento de la presente investigación.Como consecuencia de ello, el estudio de la formación de los docentes es una tarea indispensable para el mejoramiento de las prácticas pedagógicas e investigativas y la calidad de la educación en cualquier país.

Por lo tanto, este artículo es el resultado del proyecto de investigación titulado: El concepto de formación del profesor de inglés en el marco de las políticas públicas sobre formación de docentes en Colombia, desarrollado en la Unidad Central del Valle del Cauca (Uceva). El texto presenta en primer lugar una contextualización 
general y un panorama histórico de la formación de los docentes en el país. Luego, se exponen algunos proyectos y normas que se han desarrollado sobre la formación de los docentes y particularmente de los maestros de lengua extranjera en Colombia. En el siguiente aparte, se presenta una evolución del concepto de formación y posteriormente se expone, de manera detallada, el concepto de formación que se registra en las políticas públicas sobre formación en el país. Finalmente, se presentan las conclusiones y reflexión sobre dicho concepto.

\section{EL CONCEPTO DE FORMACIÓN}

Existen diversos modelos que han definido la noción de formación a través del tiempo. El concepto de formación cuenta con un carácter polisémico que puede ser comprendido desde diversos momentos históricos y perspectivas epistemológicas. El concepto de formación que se presentará a continuación, tiene su fundamento en la escuela francesa y alemana.

Sarria (2008), plantea que el concepto de formación presenta dos momentos cruciales que permiten comprender la evolución del mismo. Uno de ellos es el momento socrático donde se instala la concepción de la paideia griega, la cual designa la formación como cultura y educación del hombre, su mejoramiento y su perfeccionamiento. Esta definición de formación superpone tres grandes nociones: formación, educación y cultura. Jaeger (1997), señala que la formación en la época socrática se entendía como ideal de cultura, expresión total del hombre y de un pueblo. Así, surge la preocupación por sí mismo (sabiduría) para lograr ser mejor y lograr una transformación del ser humano. Este concepto de formación, explica Sarria (2008) no es el mismo que remite a la idea del saber técnico sino un saber reflexivo que permite una relación privilegiada entre el sujeto-saber de sí mismo y el saber sobre los objetos del mundo. El segundo momento corresponde a la llustración y remite a la consolidación del concepto de formación en su noción alemana de bildung. Fabre (2011) explica el origen de la palabra bildung a partir de su significado en alemán por los vocablos bild (imagen), vorbild (forma) y nachbild (imitación) para configurar un término que agrupa form (forma), kultur (cultura) y Aufklärung (ilustración) cuya evolución en el siglo XVIII se aleja de la concepción de forma exterior natural para acercarse a la noción de formación espiritual y cultura con los planteamientos de Herder y de Wilhelm von Humboldt.

Gadamer, por su parte, sitúa el surgimiento del concepto de bildung en la "mística medieval, la mística del barroco, su espiritualización como ascenso a la humanidad y su transformación" (Gadamer, 2001: 38). Para diversos autores alemanes como Kant, Hegel y Wilhelm von Humboldt, la formación remite a la cuestión del sujeto, conócete a ti mismo. La Bildung es "una disposición espiritual particular que el conocimiento y el sentimiento, entendidos como un acto de la totalidad del espíritu y la moralidad, producen cuando se reflejan en la sensibilidad y el carácter" (Humboldt, citado por Gadamer, 1991: 55). El concepto de bildung o formación es esencia y altura, es un modo de ser y de devenir del hombre que surge en el romanticismo alemán y cuyo origen se fundamenta en el conocimiento, la transformación y la experiencia (Zambrano, 2008).

En contraste, en la era contemporánea, la formación es definida por la transmisión de competencias, lo pragmático y lo práctico como lo expone Zambrano (2008) la formación no puede solo estar vinculada al dominio de la práctica, a la "instrumentalización" y orientación de un discurso. Ella, nos parece, tiene validez en los sujetos cuando estos pueden deducir, derivar, interpretar y comprender". De acuerdo con lo anterior, la formación implica no solo el dominio de una metodología para enseñar, sino una reflexión crítica que debe realizar el 
docente sobre lo que enseña, su relación con ese saber que enseña y las experiencias que ha vivido a partir de su relación con la disciplina que enseña. Infortunadamente, en Colombia, el concepto de formación que se pone de manifiesto en las políticas públicas sobre educación remite a la noción de capacitación y actualización tal y como se presentará en el análisis de los documentos consultados . Un acercamiento a las políticas públicas sobre formación de los docentes de lengua extranjera (inglés) permite la comprensión de cómo se ha concebido la formación desde 1980 cuando el inglés se populariza en las instituciones educativas colombianas y la formación de los maestros se enmarcaba en cursos de entrenamiento, hasta 2014 cuando se crea el Sistema Colombiano de Formación de Educadores y Lineamientos de Política y se establecen las bases para la conformación de, como su nombre lo indica, un sistema de formación que abarca la formación inicial, en servicio y avanzada.

\section{FORMACIÓN DE DOCENTES DE INGLÉS Y POLÍTICAS PÚBLICAS}

Las investigaciones sobre formación de docentes de inglés han estado centradas en el impacto de las políticas y planes sobre bilingüismo, entre ellas se destacan: Cárdenas, González y Álvarez (2010), González (2007), McNulty y Quinchía (2007), Vergara et al. (2009) y Usma (2009). De lo anterior se desprende que, a la fecha, los estudios sobre formación de docentes poco han profundizado en la naturaleza misma del concepto.

Al realizar un análisis de los procesos de formación de los docentes de inglés en Colombia, es posible plantear que estos han estado orientados al desarrollo de destrezas para enseñar la lengua extranjera, convirtiéndose en programas de actualización o capacitación como se podrá evidenciar en el análisis de los planes, proyectos y leyes nacionales sobre este particular.

Desde el siglo pasado, la enseñanza del inglés ha tomado gran relevancia en el ámbito educativo, prueba de ello son las disposiciones gubernamentales que sobre el bilingüismo se han adoptado. Sin embargo, la formación de los docentes de lengua extranjera en el país ha estado ligada a prácticas instrumentalizadoras, relacionadas más con la capacitación y el entrenamiento. Estas últimas provienen de los primeros intentos de formación que aportaron agencias internacionales como el British Council a través del COFE Project (1991). Dicho proyecto buscó formar a los docentes de inglés en aspectos metodológicos a través del uso de textos guía y mejorar los niveles de suficiencia de la lengua de los maestros. El proyecto permitió realizar cambios sustanciales en términos curriculares y didácticos pero en lo que concierne a la formación de los maestros se limitó a la preparación de los docentes en nuevas metodologías para la enseñanza de la lengua (Rubiano, Frodden \& Cardona, 2000).

\section{Enladécadadelosañosochenta, elentrenamiento} predominó como noción de formación de los docentes de lenguas extranjeras. Las formas más comunes de entrenamiento eran los cursos cortos o talleres dictados por expertos quienes seleccionaban un contenido para transmitirlo como fórmula única de éxito para la enseñanza del idioma. Algunos autores como Freeman (1989), Woodward (1997) e Imbernón (1997) conciben el entrenamiento como una técnica que da importancia al dominio de conocimientos y habilidades de una profesión o una disciplina. Lo anterior implica que el entrenamiento se limita al desarrollo de fórmulas metodológicas sobre cómo enseñar. En el mismo sentido, Richards (1989) expone las limitaciones del entrenamiento en los siguientes aspectos: el entrenamiento concibe la enseñanza desde una perspectiva atomizada y tecnicista como resultado de 
las prácticas metodológicas de los docentes, el entrenamiento no permite la reflexión del quehacer pedagógico de los docentes y se enfoca en contenidos predeterminados que impiden la reflexión de las percepciones de los docentes sobre lo que sucede al interior del aula. Además de lo anterior, durante varias décadas en el territorio colombiano, no era necesario poseer un título de licenciado ni tener suficiencia en inglés para enseñarlo.

Luego de la implementación del COFE Project y durante más de una década, la necesidad de mantener a los docentes actualizados constantemente dio paso a una nueva expresión de la formación: la capacitación. Según Zambrano (2012), después de la promulgación de la Ley General de Educación (Ley 115 de 1994), aparecen, de manera conjunta, en el escenario educativo los conceptos de capacitación y formación. La capacitación está directamente asociada con el desarrollo de habilidades para enseñar y el conocimiento de la disciplina. La capacitación de los docentes a partir de la segunda mitad de la década de los años noventa, queda establecida mediante decretos que asignan dicha responsabilidad a las Secretarías de Educación Departamentales a través de los Comités Departamentales de Capacitación. Algunas universidades también ofrecieron programas de capacitación a los profesores, los cuales eran reconocidos para el ascenso en el escalafón docente.

Con la elaboración del Plan Decenal de Educación (1995-2006), se establece un nuevo estatuto de profesionalización docente y el fortalecimiento de la formación inicial de maestros en las Escuelas Normales Superiores y el apoyo para el desarrollo de investigaciones en estas instituciones. En este plan se estipuló que para el ejercicio de la docencia se requiere poseer un título de educación superior y la profesionalización de todos los maestros vinculados con el fin de que obtengan su titulación a nivel superior. Una de las estrategias planteadas en este plan se centra en la cualificación de los docentes en términos de formación inicial y permanente.

Posteriormente, en 2004, se aprueba el Plan Nacional de Bilingüismo (PNB) como una opción para el mejoramiento de la calidad de la educación del país y en especial de la competencia comunicativa de estudiantes y docentes de lengua extranjera (inglés). Las estrategias del programa Colombia Bilingüe se fundamentaron en la formación de los maestros de inglés, la estructuración del currículo sugerido y los derechos básicos de aprendizaje del inglés, un programa de formadores nativos extranjeros y varios programas de inmersión dentro y fuera del país. Al respecto, se puede afirmar que el PNB estableció una línea de formación de los docentes de inglés que no se desarrolló pues el programa de formadores nativos extranjeros ocupó la atención y esfuerzos para lograr los objetivos propuestos en el plan.

Años más tarde, el Sistema Colombiano de Formación de Educadores y Lineamientos de Política surge de un proyecto gubernamental para el desarrollo de una política de calidad para el cuatrenio comprendido entre 2010 y 2014. Dicho documento nace de los planteamientos del Plan Nacional Decenal de Educación (20062016) sobre la necesidad de la conformación de un sistema de formación que articule los distintos niveles, centros, planes e instancias educativas.

\section{METODOLOGÍA}

Debido a la naturaleza del problema de investigación y su interés hermeneútico sobre los documentos que han configurado los procesos de formación de los docentes de lenguas extranjeras, se consideró adecuado ubicarse en un enfoque cualitativo según Creswell (2014), en la que se pudo evidenciar unas tendencias que han caracterizado las formas en las que se han orientado estos procesos. 
Esta investigación se ocupó de la descripción del concepto de formación de los docentes de inglés registrado en las políticas públicas sobre formación de maestros y bilingüismo que se han planteado entre 1980 y 2014 . El método histórico-hermenéutico permitió no solo el rastreo del concepto de formación de fuentes primarias como las leyes, decretos, planes y proyectos en el período de estudio seleccionado, sino también dio acceso a la interpretación de dicho concepto desde los entes gubernamentales nacionales. Según Aróstegui (1995) no se limita a la recopilación de antecedentes, sino que lleva al investigador a estudiar y describir el desarrollo de un fenómeno en un período de tiempo.

El objetivo del proyecto consistió en identificar cuáles son los conceptos de formación que subyacen en los lineamientos de política emanados por entes gubernamentales desde 1980 hasta 2014 sobre formación de docentes y en especial de los docentes de lenguas extranjeras. Para lograr dicho propósito, se realizó una revisión a profundidad de las normas, decretos, proyectos y planes educativos, lo anterior corresponde a un enfoque investigativo histórico con técnica de análisis documental que, de acuerdo con Martínez (2003) se define como

Un conjunto de distintas herramientas, estrategias y recursos que le permiten a un sujeto investigador obtener/construir (siempre de manera indirecta) información y conocimiento sobre algún fenómeno de la realidad (u objeto de estudio) a partir de consultar diversos tipos de documentos (esto es, a partir de acercarse a diversas "interpretaciones" preservadas en el conjunto de cierta "memoria objetivada")

Así pues, la investigación se desarrolló en las siguientes fases o etapas:

En la primera fase, también llamada heurística, se realizó la búsqueda, identificación, selección y recopilación de documentos oficiales sobre formación de docentes.

En la segunda fase se desarrolló la ordenación, análisis, clasificación y sistematización del concepto de formación extraído de los documentos consultados, en la rejilla de datos emergentes. Este instrumento según Bannister y Mair (1968) es definido como un tipo de tarea de clasificación que permite evaluar las relaciones entre constructos.

Finalmente, en la tercera fase se procedió a la triangulación de los datos a la luz de las teorías formuladas sobre el concepto de formación, así identificando unas categorías.

\section{RESULTADOS Y DISCUSIÓN}

Antes de presentar los resultados sobre el análisis del concepto de formación en los documentos oficiales consultados, se esbozarán las categorías en las cuales se han clasificado los hallazgos frente al concepto de formación como entrenamiento, capacitación o sistema en la siguiente gráfica. 


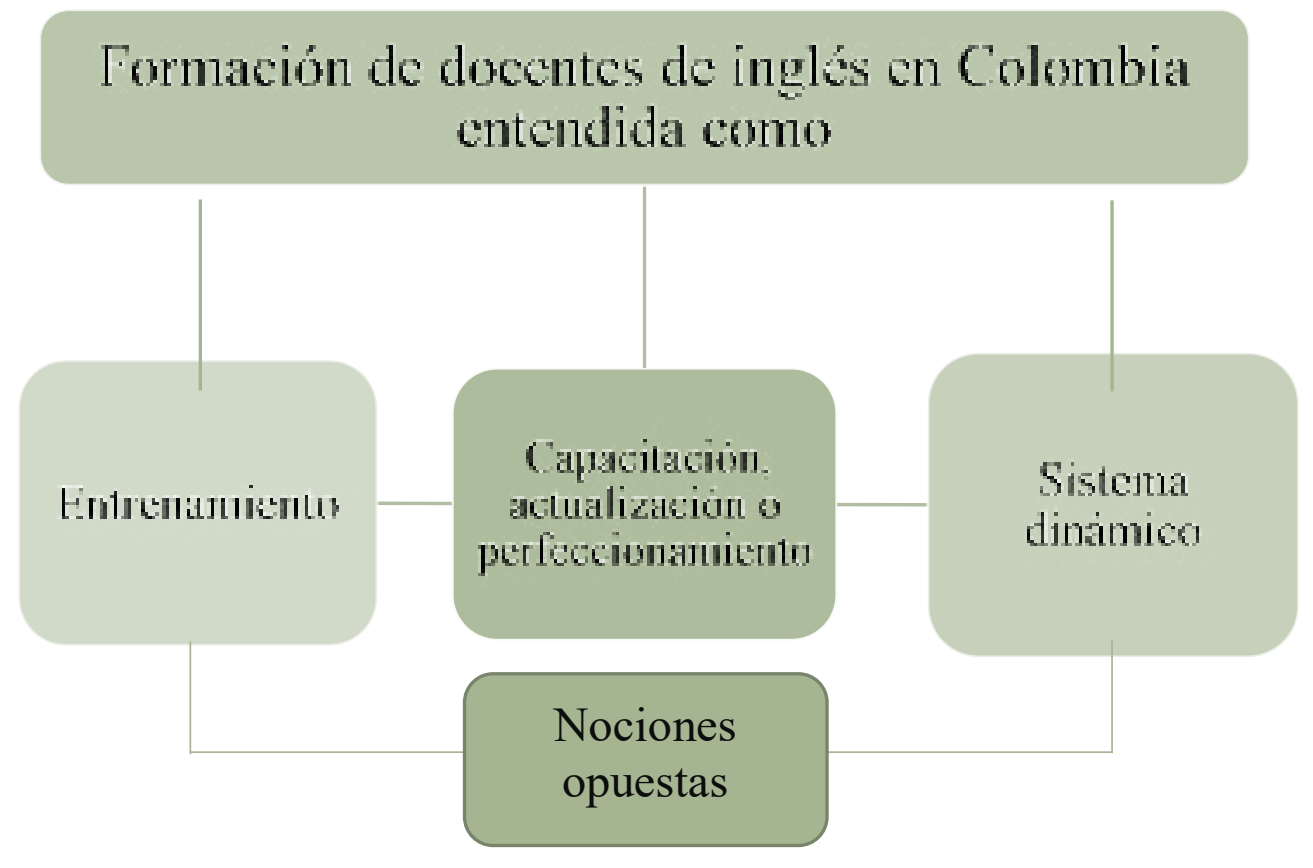

Fuente: Elaboración propia, 2019.

El concepto de formación de profesores de inglés, de acuerdo con la historia de la enseñanza de la lengua extranjera en Colombia, está directamente relacionado con el concepto de capacitación o entrenamiento tal y como se expresa en documentos oficiales que no solo cobijan a los docentes de inglés sino a los maestros de todas las áreas de conocimiento.

En primer lugar, al revisar el decreto 2277 de 1979, se evidencia en el Artículo 13 la definición de cursos de capacitación y actualización como formas de ascenso en el escalafón docente. Así, la formación se expresa en términos de cursos de capacitación. Adicionalmente, se menciona la conformación del Sistema Nacional de Capacitación para la oferta de dichos cursos.

Artículo 13. Cursos de capacitación. Los cursos de capacitación y actualización que realice el educador para ascenso serán tenidos en cuenta como créditos para obtener el título de bachiller pedagógico, licenciado en ciencias de la educación u otros en las condiciones que determine el reglamento ejecutivo. (Ministerio de Educación Nacional, 1979: 5)

En el mismo documento, el Artículo 58 refiere al Sistema Nacional de Capacitación en el cual

"La capacitación se establece como un derecho para los educadores en servicio. El Ministerio de Educación Nacional en asocio de las secretarías de educación seccionales y de las universidades oficiales, organizarán el sistema nacional de capacitación con el fin de dar cumplimiento a los objetivos señalados en el artículo anterior y garantizar a los educadores la prestación del servicio" (Ministerio de Educación Nacional, 1979: 14).

Al estudiar el documento del COFE Project, la formación de los docentes de inglés se 
presenta bajo la fórmula del entrenamiento en aspectos teóricos y prácticos con miras a lograr un cambio significativo en las prácticas de los maestros de lengua extranjera. Los objetivos trazados en dicho proyecto contemplaban el diseño e implementación de un programa de entrenamiento permanente para los docentes de las entidades asociadas en el proyecto, el cual incluiría tanto componentes teóricos como prácticos y establecer procedimientos de evaluación adecuados. Lo anterior se evidencia en el documento "A proposal for a teacher profesional development course in Colombia" como Writing Document 6, el cual plantea:

In-service teacher training as the term implies, takes a group of teachers and trains them. It gives them what to teach, when to teach, how to teach. The teachers experience is rarely taken into account. (Durán y Moreno, 1996:11).

Por su parte, el concepto de formación que se presenta en la Ley General de Educación, en el Artículo 111, la ubica en la misma línea junto con la capacitación, profesionalización y actualización a través del mejoramiento profesional, así como los programas para la formación de educadores. "Artículo 111: Profesionalización. La formación de los educadores estará dirigida a su profesionalización, actualización y perfeccionamiento hasta los más altos niveles de posgrado" (Ministerio de Educación Nacional, 1994: 23).

Nuevamente, en el Plan Decenal de Desarrollo Educativo (1996-2005), se pone en el mismo nivel a la formación y la capacitación como se expresa en el

ARTÍCULO 38. Formación y capacitación docente. La formación, capacitación, actualización y perfeccionamiento de los educadores en servicio debe contribuir de manera sustancial al mejoramiento de la calidad de la educación y a su desarrollo y crecimiento profesional, y estará dirigida especialmente a su profesionalización y especialización para lograr un mejor desempeño, mediante la actualización de conocimientos relacionados con su formación profesional, así como la adquisición de nuevas técnicas y medios que signifiquen un mejor cumplimiento de sus funciones. El Gobierno Nacional reglamentará los mecanismos, formas y alcances de la capacitación y actualización.

De igual forma, en el PNB, la formación de los docentes de lengua extranjera (inglés) se presenta como acciones puntuales para el desarrollo y/o mejoramiento de la competencia comunicativa y metodológica. Al respecto, el Plan incluye un ítem de formación docente en los siguientes aspectos: Programas de inmersión, programas de mejoramiento de la enseñanza y programas de desarrollo profesional docente.

En la misma línea, el Plan Decenal de Educación (PDE) (2005-2010) propone la cualificación de los docentes desde el nivel inicial hasta el posgrado y la conformación de un Sistema Nacional de Formación de Educadores tanto a nivel pedagógico como disciplinar. Uno de los objetivos principales del PDE fue el fortalecimiento a la formación inicial y de posgrado a través de créditos educativos.

Por el contrario, en el sistema colombiano de formación de educadores y lineamientos de política, el concepto de formación se concibe como un proceso entiende la formación como un proceso continuo, permanente e integral (saber ser, hacer y vivir con otros).

La formación docente no puede restringirse a los niveles iniciales, es un proceso continuo, a lo largo de la carrera profesional, que exige una construcción permanente e integral del educador, articulada a sujetos formadores, instituciones formadoras e instancias administrativas sociales y culturales 
determinantes. (Ministerio de Educación Nacional, 2014:46)

Por lo anterior, la formación es entonces una actividad dinámica, un proceso que cobra sentido, en la configuración general del sistema educativo (De la Garza, E., 2000), es decir, la formación de los docentes ya no se concibe como una acción aislada que tenga por objetivo el "ajuste" del perfeccionamiento, ni el entrenamiento o la actualización sino un verdadero proceso de reflexión, construcción, proyección y experiencia de los docentes. El sistema colombiano de formación de educadores y lineamientos de política, la formación no se reduce a la instrumentalización de la práctica docente, ella provoca un espacio de cuestionamiento para los maestros sobre su identidad y su papel en la construcción de una sociedad más equitativa. Al respecto, Bustamante (2006) afirma que "la formación docente entonces no puede ser una mera revisión de fórmulas didácticas o un adiestramiento en disciplinas específicas, tiene que ser el espacio que acoja la inquietud del profesor por trascender, el lugar en donde, mediante la reflexión, pueda aclarar su posición respecto de la problemática educativa, su rol en la dinámica social, su forma de entender el mundo". En el mismo sentido, (González, Villota y Ramos, 2018) confirman que la formación de un docente se evidencia en el resultado de las experiencias académicas, profesionales y de vida que permiten una transformación del sujeto y que van más allá del entrenamiento o capacitación que puede brindar un curso de actualización o perfeccionamiento.

Para finalizar, es necesario comprender las relaciones que se han instituido en estas tres categorías, ya que han movilizado los procesos de formación de los docentes de lengua extranjera (inglés) en las últimas décadas. En primer lugar, se evidenció que el concepto de formación se relaciona con el entrenamiento cuando se concibe ésta como una técnica en la que el docente a ser formado recibe instrucción de un experto y debe reproducir de manera sistemática los procedimientos enseñados. En segundo lugar, se observó que la formación se reduce a la capacitación o la actualización como una forma de renovar o perfeccionar los conocimientos adquiridos por los docentes mediante cursos de corta duración. Nuevamente se evidencia una concepción de la formación desde una perspectiva limitada que circunscribe a dicha noción a cuestiones remediales de ajuste frente al quehacer docente. Por último, la formación comprendida como un sistema dinámico abre la posibilidad de generar espacios para la reflexión y la transformación del ejercicio docente desde una nueva mirada sobre el concepto aquí estudiado.

\section{CONCLUSIONES}

El objetivo de esta investigación fue identificar cuáles son los conceptos de formación que subyacen en los lineamientos de política sobre formación de docentes de lengua extranjera (ingles). Al respecto, es posible afirmar que, luego del análisis documental realizado, se evidencia un marcado predominio de la formación entendida como entrenamiento y capacitación, actualización o perfeccionamiento desde la década de los años ochenta hasta los primeros años del siglo XXI. Lo anterior, permitió comprender que la formación de los docentes de inglés en Colombia ha estado influenciada por discursos de agencias internacionales que han permeado el sistema educativo del país. Aunque en la actualidad, y con la conformación del Sistema Colombiano de Formación de Docentes y Lineamientos de Política, se busca que la formación de los maestros se aleje de las propuestas de corto plazo como lo establecen la capacitación o el entrenamiento.

Además de esto, se encuentra que estas categorías han instituido en las diferentes instituciones educativas encargadas de la 
formación de formadores de las lenguas extranjeras, unas dinámicas ubicadas en la capacitación y actualización, pero pocas han posibilitado una reflexión pedagógica de fondo que configure una transformación de las didácticas de un segundo idioma como el inglés.

También se concluye que estas tendencias han influido en la formación docente, han generado unas acciones encaminadas a la aplicación y ejecución de contenidos temáticos sin lograr cambios profundos en el rol docente, por lo que se da apertura a investigaciones futuras que se encaminen a la búsqueda de éstas desde las prácticas del maestro y su relación con las políticas públicas.

\section{REFERENCIAS BIBLIOGRÁFICAS}

Bannister, D \& Mair, J. (1968). The evaluations of personal constructs. Academic Press: London and New York.

Bustamante Rojas, A. (2006) Educación, compromiso social y formación docente. Revista Iberoamericana de Educación-OEI-(37).

Calvo, G., Rendón Lara, D., \& Rojas García, L. (2004). Un diagnóstico de la formación docente en Colombia. Revista Colombiana De Educación, (47). https://doi. org/10.17227/01203916.5519

Cano, Granja y González (2018) The Teachers' Training in Colombia. Beginnings, Route and Current Events. Modern Applied Science; Vol. 12, No. 9; pp. 65-70.

Cárdenas, M.L., González, A., Álvarez, J. A. (2010). El desarrollo profesional de los docentes de inglés en ejercicio: algunas consideraciones conceptuales para Colombia. Folios. Segunda época. No. 31. Primer semestre de 2010. pp. 49-68.
Durán, L.M. y Moreno, L.G. (1996). Proposal for a teacher profesional development course in Colombia. Edinburg.

Fabre, M. Experiencia y Formación: la bildung. Revista Educación y Pedagogía, vol. 23, núm. 59, enero-abril, 2011.

Foucault, M. (2002) citado por Sarria, $M$ en: Significaciones y sentidos del concepto de formación y su relación con la educación superior. Revista Colombiana de Educación Superior. Vol. (1) año 0, Universidad Santiago de Cali.

Freeman, D. (1989). Teacher training, development, and decision making: a model of teaching and related strategies for language teacher education. TESOL Quarterly, 23, 1, 1989. 27-46.

Gadamer, H. (2001). Verdad y método. Salamanca: Ediciones Sígueme.

González, A. (2007). "Professional development of EFL teachers in Colombia: Between colonial and local practices". Íkala, Revista de Lenguaje y Cultura, vol. 12, No.18, pp.309-332.

González, H, Villota, J y Ramos, L. The Education of an English Professor: The Biographical Narrative. English Language Teaching; Vol. 11, No. 4; 2018, pp. 101-106.

Higueras, M. (2012), "Líneas metodológicas para la formación de profesores de lenguas extranjeras", en Revista Internacional de Lenguas Extranjeras, núm. 1, pp. 101128, http://www.revistes.publicacionsurv.cat/ index.php/rile/article/view/10/11

Imbernón, F. (1989). La formación inicial y la formación permanente del profesorado: Dos etapas de un mismo proceso. Recuperado de: http://dialnet.unirioja.es/ servlet/ articulo?codigo $=117680$ 
Imbernón, F. (1997). "La formación y el desarrollo profesional del profesorado". Hacia una nueva cultura profesional, pp. 37-60. Barcelona: Editorial Graó.

Jaegger, W. (1997). Paideia. (3ra. Reimpresión). Santafé de Bogotá: Fondo de Cultura Económica.

Martínez, J. (2003). Formato para construir referencias documentales bajo el sistema "Harvard". México: Universidad Iberoamericana. Departamento de Comunicaciones.

MC Nulty, M. \& Quinchía, D. (2007). "Designing a holistic professional development program for elementary school English teachers in Colombia". PROFILE. Issues in Teachers' Professional Development, vol. 8, pp. 131-143.

Ministerio de Educación Nacional. Decreto 080 de 1974. Recuperado de: www. mineducacion.gov.co/1759/articles-104657 archivo_pdf.pdf

Ministerio de Educación Nacional. Decreto 1278 de 2002. Recuperado de: www. mineducacion.gov.co/1621/articles-86102 archivo_pdf.pdf

Ministerio de educación Nacional. Decreto 925 de 1955. Recuperado de: http:// www.suin-juriscol.gov.co/clp/contenidos. dll/Decretos/1859596?fn=document-frame. $\mathrm{htm} \$ \mathrm{f}=$ templates $\$ 3.0$

Ministerio de Educación Nacional. Ley 115 de 1994. Recuperado de: www. mineducacion.gov.co/1621/articles-85906 archivo pdf.pdf

Ministerio de Educación Nacional. Ley General de Educación. Disponible en: https://www.mineducacion.gov.co/1621/ articles-85906_archivo_pdf.pdf
Ministerio de Educación Nacional. Plan Decenal de Educación 1996-2005. Recuperado de: $\quad$ www.mineducacion.gov.co/1621/ articles-85242 archivo_pdf.pdf

Ministerio de Educación Nacional. Plan Nacional de Bilingüismo. Disponible en: https://www.mineducacion.gov.co/1621/ articles-132560_recurso_pdf_programa_ nacional_bilinguismo.pdf

Ministerio de Educación Nacional. Plan Sectorial 2006-2010. Recuperado de: $w w w$. mineducacion.gov.co/1759/articles-152036 archivo pdf.pdf

Ministerio de Educación Nacional. Plan Sectorial 2010-2014. Disponible en: https://www. mineducacion.gov.co/1621/articles-293647_ archivo_pdf_plansectorial.pdf

Ministerio de Educación Nacional. Sistema Colombiano de Formación de Educadores y Lineamientos de Política. Recuperado de: www.mineducacion.gov. co/1759/articles-345485_anex01.pdf

Richards, J.C \& Nunan, D. (1990). Second Language Teacher Education. Cambridge, Cambridge University Press.

Richards, J.C. Conferencia: Beyond Training: Approaches to Teacher Education in Language Teaching. Second Language Teacher Education, Macquarie University, Sydney, 15 June, 1989.

Rubiano, C. I., Frodden, C. \& Cardona, G. (2000). The impact of the colombian framework for english (COFE) project: an insiders'perspective. Íkala. 5 (1-2), pp. $37-54$.

Sáez-Rosenkranz, I. (2016). El método histórico aplicado a la investigación educativa. REIRE. Revista d'Innovació i Recerca en Educació, 9 (2), 106-113. Doi: 10.1344/ reire2016.9.2927. 
Sarria, M. (2008). Significaciones y sentidos del concepto de formación y su relación con la Educación Superior. Revista Colombiana de Educación Superior. Vol 1, 8-25.

Usma, J. A. (2009). "Education and language policy in Colombia: Exploring processes of Inclusion, Exclusion, and Stratification in Times of Global Reform". PROFILE, Issues in Teachers' Professional Development, vol. 11, pp. 123-142.

Vergara, O., Hernández, F. \& Cárdenas, R. (2009). "Classroom Research and Professional Development". PROFILE. Issues in a Teachers' Professional Developtment, vol. 11, pp. 169-191.

Vierhaus, R. (2002). Formación (Bildung). Separata Revista Educación y Pedagogía, Universidad de Antioquia- Facultad de Educación, Mayo-Agosto de 2002.

Woodward, T. (1991). Models and metaphors in language teacher training. Loop input and other suggestions. Cambridge: Cambridge University Press.

Zambrano, A. (2001). Pedagogía, Educabilidad y Formación de Docentes. Editorial Magisterio, Bogotá.

Zambrano, A. (2008). Formación y autonomía: tensiones en las modalidades académicas típicas. Revista Colombiana de Educación Superior. Vol 1, 43-59.

Zambrano, A. Formación docente en Colombia. Arqueología de la política pública y del escenario internacional. Educere, vol. 16, núm. 54, mayo-agosto, 2012, pp. 11-19. 